\title{
Communication
}

\section{Existence Results for Fractional Neutral Functional Differential Equations with Random Impulses}

\author{
Annamalai Anguraj ${ }^{1}$, Mullarithodi C. Ranjini ${ }^{2}$, Margarita Rivero ${ }^{3}$ and Juan J. Trujillo $^{4, *}$ \\ ${ }^{1}$ Department of Mathematics, PSG College of Arts and Science, Coimbatore-641 014, Tamil Nadu, \\ India; E-Mail: angurajpsg@yahoo.com \\ ${ }^{2}$ Department of Mathematics, Karunya University, Coimbatore-641 114, Tamil Nadu, India; \\ E-Mail: ranjiniprasad@gmail.com \\ 3 Departamento de Análisis Matemático, Universidad de La Laguna, 38271 La Laguna, Tenerife, \\ Spain; E-Mail: mrivero@ull.es \\ 4 Departamento de Matemáticas, Universidad de La Laguna, Estadística, e I.O., 38271 La Laguna, \\ Tenerife, Spain \\ * Author to whom correspondence should be addressed; E-Mail: jtrujill@ullmat.es; \\ Tel.: +34-922318209.
}

Academic Editor: Hari M. Srivastava

Received: 8 December 2014 / Accepted: 19 January 2015 / Published: 21 January 2015

\begin{abstract}
In this paper, we investigate the existence of solutions for the fractional neutral differential equations with random impulses. The results are obtained by using Krasnoselskii's fixed point theorem. Examples are added to show applications of the main results.
\end{abstract}

Keywords: fractional neutral differential equations; random impulses; existence; fixed point theorem

\section{Introduction}

Fractional Differential Equations, in which an unknown function is contained under the operation of a derivative of fractional order, have been of great interest recently. Many papers and books on fractional differential equations have appeared (see [1-6]). In [7], Lakshmikantham and Vatsala derived the basic theory of fractional differential equations. In [8], Hernandez et al. proved the existence of solutions of abstract fractional differential equations by using fixed point techniques. 
On the other hand, impulsive differential systems are proved to be adequate mathematical models for numerous processes and phenomena studied in population dynamics, physics, chemistry and engineering. In recent years, some impressive results have been obtained in this area $($ see $[7,9])$. For the general theory of impulsive differential systems, the reader can refer to [10].

However, actual impulses do not always happen at fixed points but usually at random points. When the impulses exist at random points, the solutions of the differential systems are stochastic processes. Random impulsive systems are more realistic than deterministic impulsive systems. The study of random impulsive differential equations is a new area of research. So far, few results have been discussed in random impulsive systems. The existence and uniqueness of differential system with random impulses is studied by Anguraj et al. in [11,12]. In [13], Wu and Duan discussed the oscillation, stability and boundedness of second-order differential systems with random impulses, and in $[14,15]$, the authors proved the existence and stability results of random impulsive semilinear differential systems.

Recently, the study of impulsive differential equations has attracted a great deal of attention in fractional dynamics and its theory has been treated in several works (see [16,17]). Also, several authors [18-20] have studied the behaviour of neutral dfferential equations. The main reason for this interest is that delay differential equations play an important role in applications. For instance, in biological applications, delay equations give a better description of fluctuations in population than the ordinary ones. Also, neutral delay differential equations appear as models of electrical networks which contain lossless transmission lines. Such networks arise, for example, in high speed computers where lossless transmission lines are used to interconnect switching circuits. In [21], Agarwal, Zhou and He proved the existence results of fractional neutral functional differential equations and also in [22], Anguraj et al. proved the existence results for fractional impulsive neutral differential equations. By the motivation of the recent surge in developing the theory of fractional neutral differential equations, we present a new idea of research to prove the existence of fractional neutral differential equations with random impulses.

This paper is divided into four sections. In Secion 2, we recall some basic definitions and preliminary facts. In Section 3, we shall establish the existence theorem for the Equation (1) by using the Krasnoselskii's fixed point theorem and in the final section, an illustrative example is presented.

\section{Preliminaries}

Let $R^{n}$ be the $n$-dimensional Euclidean space and $\Omega$ a non-empty set. Assume that $\tau_{k}$ is a random variable defined from $\Omega$ to $D_{k} \stackrel{\text { def. }}{=}\left(0, d_{k}\right)$ for all $k=1,2, \ldots$ where $0<d_{k}<\infty$. Furthermore, assume that $\tau_{i}$ and $\tau_{j}$ are independent of each other as $i \neq j$ for $i, j=1,2, \ldots$. Let $\tau, T \in R$ be two constants satisfying $\tau<T$. We denote $R_{\tau}=[\tau, T], R^{+}=\left[t_{0}, \infty\right)$.

We consider the fractional neutral functional differential equations with random impulses of the form:

$$
\left\{\begin{array}{l}
{ }^{c} D^{\alpha}\left(x(t)-g\left(t, x_{t}\right)\right)=A(t, x) x(t)+f\left(t, x_{t}\right), \quad t \in[\tau, T], t \neq \zeta_{k} \\
x\left(\zeta_{k}\right)=b_{k}\left(\tau_{k}\right) x\left(\zeta_{k}^{-}\right), \\
x_{t_{0}}=\phi
\end{array}\right.
$$


where ${ }^{c} D^{\alpha}$ is the standard Caputo's fractional derivative of order $0<\alpha<1$. f,g: $R_{\tau} \times C \rightarrow R^{n}$, $C=C\left([-r, 0], R^{n}\right)$ are given functions mapping $[-r, 0]$ into $R^{n}$ with some given $r>0 . \phi$ is a function defined from $[-r, 0]$ to $R^{n} ; x_{t}$ is a function when $t$ is fixed, defined by $x_{t}(\theta)=x(t+\theta)$, for $\theta \in[-r, 0] ; \zeta_{0}=t_{0}$ and $\zeta_{k}=\zeta_{k-1}+\tau_{k}$ for $k=1,2, \ldots$. Here $t_{0} \in R_{\tau}$ is an arbitrary given real number. Obviously, $t_{0}=\zeta_{0}<\zeta_{1}<\zeta_{2}<\ldots \ldots<\zeta_{k}<\ldots ; b_{k}: D_{k} \rightarrow R^{n \times n}$ is a matrix valued function for each $k=1,2, \ldots, x\left(\zeta_{k}^{-}\right)=\lim _{t \rightarrow \zeta_{k}} x(t)$ with the norm $\|x\|_{t}=\sup _{t-r<s<t}\|x(s)\|$ for each $t$ satisfying $\tau \leq t \leq T$ and $T \in R^{+}$is a given number, $\|$.$\| is any given norm in R^{n}$. Let $\mathcal{B}\left(R^{n}\right)$ denote the Banach space of bounded linear operators from $R^{n}$ to $R^{n}$ with the norm $\|A\|_{\mathcal{B}\left(R^{n}\right)}=\sup \{\|A(y)\|:\|y\|=1\}$.

Denote $\left\{B_{t}, t \geq 0\right\}$ the simple counting process generated by $\zeta_{n}$, that is, $\left\{B_{t} \geq n\right\}=\left\{\zeta_{n} \leq t\right\}$, and denote $\mathcal{F}_{t}$ the $\sigma$-algebra generated by $\left\{B_{t}, t \geq 0\right\}$. Then $\left(\Omega, P,\left\{\mathcal{F}_{t}\right\}\right)$ is a probability space. For the simplicity, denote the Banach space $\Gamma=\left\{\right.$ all functions defined from $\left[t_{0}-r, \infty\right)$ to $R^{n}$ with the norm defined by $\left.\|\chi\|_{\Gamma}=\sup _{t \geq t_{0}} E\|\chi\|_{t}\right\}$.

Definition 1. ([4]). The fractional integral of order $q$ with the lower limit $t_{0}$ for a function $f$ is defined as

$$
I^{q} f(t)=\frac{1}{\Gamma(q)} \int_{t_{0}}^{t}(t-s)^{(q-1)} f(s) d s, \quad t>t_{0}, \quad q>0
$$

provided the right-hand side is pointwise defined on $\left[t_{0}, \infty\right)$, where $\Gamma$ is the gamma function.

Definition 2. ([4]). Riemann-Liouville $(R-L)$ derivative of order $q$ with the lower limit $t_{0}$ for a function $f:\left[t_{0}, \infty\right) \longrightarrow R$ can be written as

$$
D^{q} f(t)=\frac{1}{\Gamma(n-q)} \frac{d^{n}}{d t^{n}} \int_{t_{0}}^{t}(t-s)^{(n-q-1)} f(s) d s, \quad t>t_{0}, \quad n-1<q<n .
$$

The most important property of $R$ - $L$ fractional derivative is that for $t>t_{0}$ and $q>0$, we have $D^{q}\left(I^{q} f(t)\right)=f(t)$, which means that $R$-L fractional differentiation operator is a left inverse to the $R$ - $L$ fractional integration operator of the same order $q$.

Definition 3. ([4]). The Caputo fractional derivative of order $q$ with the lower limit $t_{0}$ for a function $f:\left[t_{0}, \infty\right) \longrightarrow R$ can be written as

$$
{ }^{c} D^{q} f(t)=\frac{1}{\Gamma(n-q)} \int_{t_{0}}^{t}(t-s)^{(n-q-1)} f^{(n)}(s) d s=I^{(n-q)} f^{(n)}(t), t>t_{0}, n-1<q<n .
$$

Obviously, Caputo's derivative of a constant is equal to zero.

We shall state some properties of the operators $I^{\alpha}$ and ${ }^{c} D^{\alpha}$.

Proposition 4. ([4,15]) For $\alpha, \beta>o$ and $f$ as a suitable function, we have

(i) $I^{\alpha} I^{\beta} f(t)=I^{\alpha+\beta} f(t)$

(ii) $I^{\alpha} I^{\beta} f(t)=I^{\beta} I^{\alpha} f(t)$

(iii) $I^{\alpha}(f(t)+g(t))=I^{\alpha} f(t)+I^{\alpha} g(t)$ 
(iv) $I^{\alpha c} D^{\alpha} f(t)=f(t)-f(0), 0<\alpha<1$

(v) ${ }^{c} D^{\alpha} I^{\alpha} f(t)=f(t)$

(vi) ${ }^{c} D^{\alpha} f(t)=I^{(1-\alpha)} D f(t)=I^{(1-\alpha)} f^{\prime}(t), 0<\alpha<1, D=\frac{d}{d t}$

(vii) ${ }^{c} D^{\alpha c} D^{\beta} f(t) \neq{ }^{c} D^{(\alpha+\beta)} f(t)$

(ix) ${ }^{c} D^{\alpha c} D^{\beta} f(t) \neq{ }^{c} D^{\beta}{ }^{c} D^{\alpha} f(t)$

In [7], Balachandran and Trujillo observed that both the R-L and the Caputo fractional differential operators do not possess neither semigroup nor commutative properties, which are inherent to the derivatives on integer order. For basic facts about fractional integrals and fractional derivatives one can refer to the books $[4,6,9]$.

Definition 5. For a given $T \in\left(t_{0}, \infty\right)$, a stochastic process $\left\{x(t), t_{0}-r \leq t \leq T\right\}$ is called a solution to the Equation (1) in $\left(\Omega, P,\left\{\mathcal{F}_{t}\right\}\right)$, if

(i) $x(t)$ is $\mathcal{F}_{t}$-adapted.

(ii) $x\left(t_{0}+s\right)=\phi(s)$ when $s \in[-r, 0]$, and

$$
\begin{aligned}
x(t)= & g\left(t, x_{t}\right)+\sum_{k=0}^{\infty}\left[\sum_{i=1}^{k} g\left(t_{i}, x_{t_{i}}\right)\left(b_{i}\left(\tau_{i}\right)-1\right) \prod_{j=i+1}^{k} b_{j}\left(\tau_{j}\right)+\prod_{i=1}^{k} b_{i}\left(\tau_{i}\right)\left[\phi(0)-g\left(t_{0}, \phi\right)\right]\right. \\
& +\sum_{i=1}^{k} \prod_{j=i}^{k} \frac{b_{j}\left(\tau_{j}\right)}{\Gamma(\alpha)}\left\{\int_{\zeta_{i-1}}^{\zeta_{i}}\left(\zeta_{i}-s\right)^{\alpha-1} A(s, x) x(s) d s\right\} \\
& +\frac{1}{\Gamma(\alpha)} \int_{\zeta_{k}}^{t}(t-s)^{\alpha-1} A(s, x) x(s) d s \\
& +\sum_{i=1}^{k} \prod_{j=i}^{k} \frac{b_{j}\left(\tau_{j}\right)}{\Gamma(\alpha)}\left\{\int_{\zeta_{i-1}}^{\zeta_{i}}\left(\zeta_{i}-s\right)^{\alpha-1} f\left(s, x_{s}\right) d s\right\} \\
& \left.+\frac{1}{\Gamma(\alpha)} \int_{\zeta_{k}}^{t}(t-s)^{\alpha-1} f\left(s, x_{s}\right) d s\right] I_{\left[\zeta_{k}, \zeta_{k+1}\right)}(t), \quad t \in\left[t_{0}, T\right]
\end{aligned}
$$

where $\prod_{j=i}^{k} b_{j}\left(\tau_{j}\right)=b_{k}\left(\tau_{k}\right) b_{k-1}\left(\tau_{k-1}\right) \ldots b_{i}\left(\tau_{i}\right), \prod_{j=m}^{n}()=$.1 as $m>n$ and $I_{A}($.$) is the index$ function, i.e.,

$$
I_{A}(t)= \begin{cases}1, & \text { if } t \in A \\ 0, & \text { if } t \notin A\end{cases}
$$

Lemma 6. (Krasnoselskii's Fixed point theorem). Let $X$ be a Banach space, let E be a bounded closed convex subset of $X$ and let $S, U$ be maps of $E$ into $X$ such that $S x+U y \in E$ for every pair $x, y \in E$. If $S$ is a contraction and $U$ is Completely continuous, then the equation $S x+U x=x$ has a solution on $E$. 


\section{Existence Results}

In this section, we discuss the existence of the solutions of the system (1). Before stating and proving the main results, we introduce the following hypothesis.

$\left(H_{1}\right)$ The function $f$ satisfies the Lipschitz condition and there exists a positive constant $L_{1}>0$ such that for $x, y \in C$ and $t \in[\tau, T]$,

$$
\left\|f\left(t, x_{t}\right)-f\left(t, y_{t}\right)\right\| \leq L_{1}\|x-y\| .
$$

$\left(H_{2}\right)$ The function $g$ is continuous and there exists a constant $L_{2}>0$ such that

$$
\left\|g\left(t, x_{t}\right)\right\| \leq L_{2}
$$

$\left(H_{3}\right) A: J \times R^{n} \rightarrow \mathcal{B}\left(R^{n}\right)$ is a continuous bounded linear operator and there exists a constant $L_{3}>0$ such that

$$
\|A(t, x)-A(t, y)\| \leq L_{3}\|x-y\|
$$

for all $x, y \in R^{n}$.

$\left(H_{4}\right)$ The functions $f$ and $A$ are continuous and there exist a non-negative constant $k$ such that

$$
\begin{aligned}
& \|f(t, 0)\| \leq k,\left\|f\left(t, x_{t}\right)\right\| \leq L_{1}\|x\|+k \\
& \|A(t, 0)\| \leq k,\|A(t, x)\| \leq L_{3}\|x\|+k .
\end{aligned}
$$

$\left(H_{5}\right) \max _{i, k}\left\{\prod_{j=i}^{k}\left\|b_{j}\left(\tau_{j}\right)\right\|\right\}$ is uniformly bounded. (i.e.) there is a $B>0$ such that

$$
\max _{i, k}\left\{\prod_{j=i}^{k}\left\|b_{j}\left(\tau_{j}\right)\right\|\right\} \leq B, \forall \tau_{j} \in D_{j}, j=1,2, \ldots
$$

$\left(H_{6}\right)$ There exists a constant $N>0$ such that

$$
\max _{k}\left\{\left\|g\left(t_{i}, x_{t_{i}}\right)\left(b_{i}\left(\tau_{i}\right)-1\right)\right\|\right\} \leq N
$$

Theorem 7. Under the hypotheses $\left(H_{1}\right)-\left(H_{6}\right)$, there exists a solution for the equation $(1)$ if

$$
\frac{\left(T-t_{0}\right)^{\alpha}}{\Gamma(\alpha+1)} \max \{1, B\}\left[\left(L_{3} r+k\right) r+\left(L_{1} r+k\right)\right]+L_{2}+B\left(N+E\left\|\phi(0)-g\left(t_{0}, \phi\right)\right\|\right) \leq r
$$

and

$$
\frac{\left(T-t_{0}\right)^{\alpha}}{\Gamma(\alpha+1)} \max \{1, B\}\left[2 L_{3} r+k+L_{1}\right]<1
$$


Proof: Let $T$ be an arbitrary positive number $t_{0}<T<\infty$. Let us define an operator $P: \Gamma \rightarrow \Gamma$ as follows:

$$
P x(t)=\phi\left(t-t_{0}\right), \quad t \in\left[t_{0}-r, t_{0}\right)
$$

and

$$
\begin{aligned}
P x(t)= & g\left(t, x_{t}\right)+\sum_{k=0}^{\infty}\left[\sum_{i=1}^{k} g\left(t_{i}, x_{t_{i}}\right)\left(b_{i}\left(\tau_{i}\right)-1\right) \prod_{j=i+1}^{k} b_{j}\left(\tau_{j}\right)+\prod_{i=1}^{k} b_{i}\left(\tau_{i}\right)\left[\phi(0)-g\left(t_{0}, \phi\right)\right]\right. \\
& +\sum_{i=1}^{k} \prod_{j=i}^{k} \frac{b_{j}\left(\tau_{j}\right)}{\Gamma(\alpha)}\left\{\int_{\zeta_{i-1}}^{\zeta_{i}}\left(\zeta_{i}-s\right)^{\alpha-1} A(s, x) x(s) d s\right\} \\
& +\frac{1}{\Gamma(\alpha)} \int_{\zeta_{k}}^{t}(t-s)^{\alpha-1} A(s, x) x(s) d s \\
& +\sum_{i=1}^{k} \prod_{j=i}^{k} \frac{b_{j}\left(\tau_{j}\right)}{\Gamma(\alpha)}\left\{\int_{\zeta_{i-1}}^{\zeta_{i}}\left(\zeta_{i}-s\right)^{\alpha-1} f\left(s, x_{s}\right) d s\right\} \\
& \left.+\frac{1}{\Gamma(\alpha)} \int_{\zeta_{k}}^{t}(t-s)^{\alpha-1} f\left(s, x_{s}\right) d s\right] I_{\left[\zeta_{k}, \zeta_{k+1}\right)}(t), t \in\left[t_{0}, T\right]
\end{aligned}
$$

Let $B_{r}=\{x \in \Gamma:\|x\| \leq r\}$

We define the operators $S$ and $U$ on $B_{r}$ as

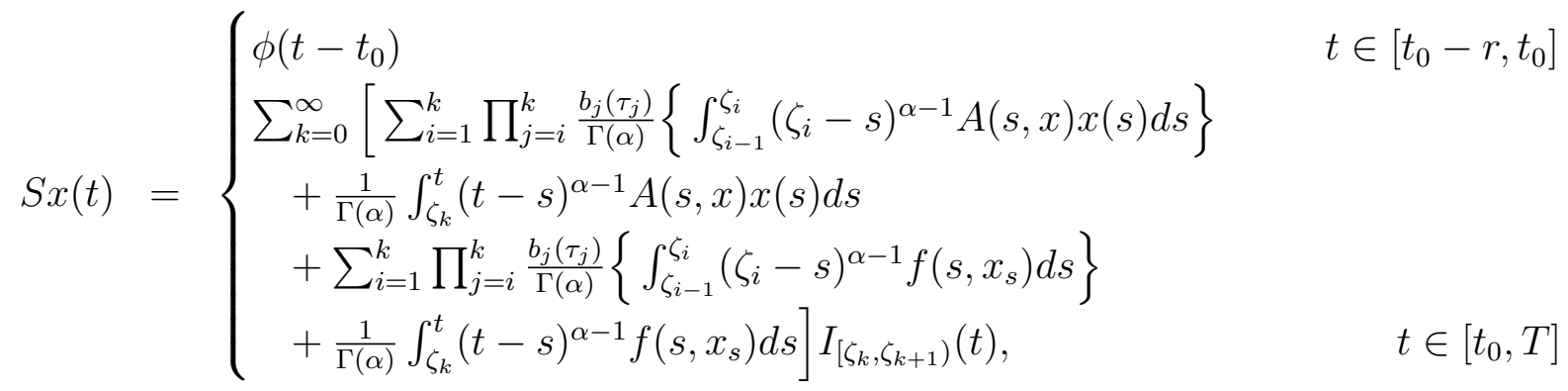

and

$$
U x(t)=\left\{\begin{array}{lr}
\phi\left(t-t_{0}\right) & t \in\left[t_{0}-r, t_{0}\right] \\
g\left(t, x_{t}\right)+\sum_{k=0}^{\infty}\left[\sum_{i=1}^{k} g\left(t_{i}, x_{t_{i}}\right)\left(b_{i}\left(\tau_{i}\right)-1\right) \prod_{j=i+1}^{k} b_{j}\left(\tau_{j}\right)\right. & \\
\left.+\prod_{i=1}^{k} b_{i}\left(\tau_{i}\right)\left[\phi(0)-g\left(t_{0}, \phi\right)\right]\right] I_{\left[\zeta_{k}, \zeta_{k+1}\right)}(t), & t \in\left[t_{0}, T\right]
\end{array}\right.
$$

Next, we have to prove that $S+U$ has a fixed point in $B_{r}$.

The proof is divided into three steps.

Step I: To prove $S x+U y \in B_{r}$, for all $x, y \in B_{r}$.

For $x, y \in B_{r}$, consider,

$$
\begin{aligned}
\|S x+U y\|= & \| \sum_{k=0}^{\infty}\left[\sum_{i=1}^{k} \prod_{j=i}^{k} \frac{b_{j}\left(\tau_{j}\right)}{\Gamma(\alpha)} \int_{\zeta_{i-1}}^{\zeta_{i}}\left(\zeta_{i}-s\right)^{\alpha-1} A(s, x) x(s) d s\right. \\
& +\frac{1}{\Gamma(\alpha)} \int_{\zeta_{k}}^{t}(t-s)^{\alpha-1} A(s, x) x(s) d s+\sum_{i=1}^{k} \prod_{j=i}^{k} \frac{b_{j}\left(\tau_{j}\right)}{\Gamma(\alpha)} \int_{\zeta_{i-1}}^{\zeta_{i}}\left(\zeta_{i}-s\right)^{\alpha-1} f\left(s, x_{s}\right) d s \\
& \left.+\frac{1}{\Gamma(\alpha)} \int_{\zeta_{k}}^{t}(t-s)^{\alpha-1} f\left(s, x_{s}\right) d s\right] I_{\left[\zeta_{k}, \zeta_{k+1}\right)}(t)
\end{aligned}
$$




$$
\begin{aligned}
& +g\left(t, y_{t}\right)+\sum_{k=0}^{\infty}\left[\sum_{i=1}^{k} g\left(t_{i}, y_{t_{i}}\right)\left(b_{i}\left(\tau_{i}\right)-1\right) \prod_{j=i+1}^{k} b_{j}\left(\tau_{j}\right)\right. \\
& \left.+\prod_{i=1}^{k} b_{i}\left(\tau_{i}\right)\left[\phi(0)-g\left(t_{0}, \phi\right)\right]\right] I_{\left[\zeta_{k}, \zeta_{k+1}\right)}(t) \| \\
& \leq\left\|g\left(t, y_{t}\right)\right\|+\sum_{k=0}^{\infty}\left[\sum_{i=1}^{k}\left\|g\left(t_{i}, y_{t_{i}}\right)\left(b_{i}\left(\tau_{i}\right)-1\right)\right\| \prod_{j=i+1}^{k}\left\|b_{j}\left(\tau_{j}\right)\right\|\right. \\
& \left.+\prod_{i=1}^{k}\left\|b_{i}\left(\tau_{i}\right)\right\|\left\|\phi(0)-g\left(t_{0}, \phi\right)\right\|\right] I_{\left[\zeta_{k}, \zeta_{k+1}\right)}(t) \\
& +\sum_{k=0}^{\infty}\left[\sum_{i=1}^{k} \prod_{j=i}^{k} \frac{\left\|b_{j}\left(\tau_{j}\right)\right\|}{\Gamma(\alpha)} \int_{\zeta_{i-1}}^{\zeta_{i}}\left(\zeta_{i}-s\right)^{\alpha-1}\|A(s, x)\|\|x(s)\| d s\right. \\
& \left.+\frac{1}{\Gamma(\alpha)} \int_{\zeta_{k}}^{t}(t-s)^{\alpha-1}\|A(s, x)\|\|x(s)\| d s\right] I_{\left[\zeta_{k}, \zeta_{k+1}\right)}(t) \\
& +\sum_{k=0}^{\infty}\left[\sum_{i=1}^{k} \prod_{j=i}^{k} \frac{\left\|b_{j}\left(\tau_{j}\right)\right\|}{\Gamma(\alpha)} \int_{\zeta_{i-1}}^{\zeta_{i}}\left(\zeta_{i}-s\right)^{\alpha-1}\left\|f\left(s, x_{s}\right)\right\| d s\right. \\
& \left.+\frac{1}{\Gamma(\alpha)} \int_{\zeta_{k}}^{t}(t-s)^{\alpha-1}\left\|f\left(s, x_{s}\right)\right\| d s\right] I_{\left[\zeta_{k}, \zeta_{k+1}\right)}(t) \\
& \leq L_{2}+\max _{k}\left\{\sum_{i=1}^{k}\left\|g\left(t_{i}, y_{t_{i}}\right)\left[b_{i}\left(\tau_{i}\right)-1\right]\right\|\right\} \max _{i, k}\left\{\prod_{j=i+1}^{k}\left\|b_{j}\left(\tau_{j}\right)\right\|\right\} \\
& +\max _{k}\left\{\prod_{i=1}^{k}\left\|b_{i}\left(\tau_{i}\right)\right\|\right\}\left\|\phi(0)-g\left(t_{0}, \phi\right)\right\| \\
& +\frac{1}{\Gamma(\alpha)} \max _{i, k}\left\{1, \prod_{j=i}^{k}\left\|b_{j}\left(\tau_{j}\right)\right\|\right\} \int_{t_{0}}^{t}(t-s)^{\alpha-1}\|A(s, x)\|\|x(s)\| d s \\
& +\frac{1}{\Gamma(\alpha)} \max _{i, k}\left\{1, \prod_{j=i}^{k}\left\|b_{j}\left(\tau_{j}\right)\right\|\right\} \int_{t_{0}}^{t}(t-s)^{\alpha-1}\left\|f\left(s, x_{s}\right)\right\| d s \\
& \leq L_{2}+N B+B\left\|\phi(0)-g\left(t_{0}, \phi\right)\right\| \\
& +\frac{1}{\Gamma(\alpha)} \max _{i, k}\{1, B\} \int_{t_{0}}^{t}(t-s)^{\alpha-1}[\|A(s, x)-A(s, 0)\|+\|A(s, 0)\|]\|x(s)\| d s \\
& +\frac{1}{\Gamma(\alpha)} \max _{i, k}\{1, B\} \int_{t_{0}}^{t}(t-s)^{\alpha-1}\left[\left\|f\left(s, x_{s}\right)-f(s, 0)\right\|+\|f(s, 0)\|\right] d s \\
& \leq L_{2}+B\left(N+\left\|\phi(0)-g\left(t_{0}, \phi\right)\right\|\right) \\
& +\frac{1}{\Gamma(\alpha)} \max _{i, k}\{1, B\} \int_{t_{0}}^{t}(t-s)^{\alpha-1}\left[L_{3}\|x\|+k\right]\|x(s)\| d s \\
& +\frac{1}{\Gamma(\alpha)} \max _{i, k}\{1, B\} \int_{t_{0}}^{t}(t-s)^{\alpha-1}\left[L_{1}\|x\|+k\right] d s
\end{aligned}
$$


Now,

$$
\begin{aligned}
E\|(S x+U y)(t)\| \leq & L_{2}+B\left(N+E\left\|\phi(0)-g\left(t_{0}, \phi\right)\right\|\right) \\
& +\frac{1}{\Gamma(\alpha)} \max _{i, k}\{1, B\} \int_{t_{0}}^{t}(t-s)^{\alpha-1}\left[L_{3} E\|x\|+k\right] E\|x(s)\| d s \\
& +\frac{1}{\Gamma(\alpha)} \max _{i, k}\{1, B\} \int_{t_{0}}^{t}(t-s)^{\alpha-1}\left[L_{1} E\|x\|+k\right] d s
\end{aligned}
$$

$\sup _{t_{0} \leq t \leq T} E\|(S x+U y)(t)\| \leq L_{2}+B\left(N+\sup _{t_{0} \leq t \leq T} E\left\|\phi(0)-g\left(t_{0}, \phi\right)\right\|\right)$

$$
\begin{aligned}
& +\frac{1}{\Gamma(\alpha)} \max _{i, k}\{1, B\} \int_{t_{0}}^{t}(t-s)^{\alpha-1}\left[L_{3} \sup _{t_{0} \leq t \leq T} E\|x\|+k\right] \sup _{t_{0} \leq t \leq T} E\|x(s)\| d s \\
& +\frac{1}{\Gamma(\alpha)} \max _{i, k}\{1, B\} \int_{t_{0}}^{t}(t-s)^{\alpha-1}\left[L_{1} \sup _{t_{0} \leq t \leq T} E\|x\|+k\right] d s \\
\leq & L_{2}+B\left(N+\left\|\phi(0)-g\left(t_{0}, \phi\right)\right\|\right) \\
& +\frac{\left(T-t_{0}\right)^{\alpha}}{\Gamma(\alpha+1)} \max \{1, B\}\left[\left(L_{3} r+k\right) r+\left(L_{1} r+k\right)\right]
\end{aligned}
$$

Therefore, by Equation (3) $\|S x+U y\|=\sup _{t \geq t_{0}} E\|S x+U y\| \leq r$, which means that $S x+U y \in B_{r}$.

Step II: To prove $S$ is a contraction on $B_{r}$.

Let $x, y \in B_{r}$.

\section{Consider,}

$$
\begin{aligned}
S x(t)-S y(t)= & \sum_{k=0}^{\infty}\left[\sum_{i=1}^{k} \prod_{j=i}^{k} \frac{b_{j}\left(\tau_{j}\right)}{\Gamma(\alpha)} \int_{\zeta_{i-1}}^{\zeta_{i}}\left(\zeta_{i}-s\right)^{\alpha-1}[A(s, x) x(s)-A(s, y) y(s)] d s\right. \\
& +\frac{1}{\Gamma(\alpha)} \int_{\zeta_{k}}^{t}(t-s)^{\alpha-1}[A(s, x) x(s)-A(s, y) y(s)] d s \\
& +\sum_{i=1}^{k} \prod_{j=i}^{k} \frac{b_{j}\left(\tau_{j}\right)}{\Gamma(\alpha)} \int_{\zeta_{i-1}}^{\zeta_{i}}\left(\zeta_{i}-s\right)^{\alpha-1}\left[f\left(s, x_{s}\right)-f\left(s, y_{s}\right)\right] d s \\
& \left.+\frac{1}{\Gamma(\alpha)} \int_{\zeta_{k}}^{t}(t-s)^{\alpha-1}\left[f\left(s, x_{s}\right)-f\left(s, y_{s}\right)\right] d s\right] I_{\left[\zeta_{k}, \zeta_{k+1}\right)}(t) .
\end{aligned}
$$

Then,

$$
\begin{aligned}
& \|S x(t)-S y(t)\| \\
& \leq \sum_{k=0}^{\infty}\left[\sum_{i=1}^{k} \prod_{j=i}^{k} \frac{\left\|b_{j}\left(\tau_{j}\right)\right\|}{\Gamma(\alpha)} \int_{\zeta_{i-1}}^{\zeta_{i}}\left(\zeta_{i}-s\right)^{\alpha-1}[\|A(s, x)(x(s)-y(s))\|+\|(A(s, x)-A(s, y)) y(s)\|] d s\right. \\
& \left.\quad+\frac{1}{\Gamma(\alpha)} \int_{\zeta_{k}}^{t}(t-s)^{\alpha-1}[\|A(s, x)(x(s)-y(s))\|+\|(A(s, x)-A(s, y)) y(s)\|] d s\right] I_{\left[\zeta_{k}, \zeta_{k+1}\right)}(t)
\end{aligned}
$$




$$
\begin{aligned}
& +\sum_{k=0}^{\infty}\left[\sum_{i=1}^{k} \prod_{j=i}^{k} \frac{\left\|b_{j}\left(\tau_{j}\right)\right\|}{\Gamma(\alpha)} \int_{\zeta_{i-1}}^{\zeta_{i}}\left(\zeta_{i}-s\right)^{\alpha-1}\left[\left\|f\left(s, x_{s}\right)-f\left(s, y_{s}\right)\right\|\right] d s\right. \\
& \left.+\frac{1}{\Gamma(\alpha)} \int_{\zeta_{k}}^{t}(t-s)^{\alpha-1}\left[\left\|f\left(s, x_{s}\right)-f\left(s, y_{s}\right)\right\|\right] d s\right] I_{\left[\zeta_{k}, \zeta_{k+1}\right)}(t) \\
\leq & \frac{1}{\Gamma(\alpha)} \max _{i, k}\left\{1, \prod_{j=i}^{k}\left\|b_{j}\left(\tau_{j}\right)\right\|\right\} \int_{t_{0}}^{t}(t-s)^{\alpha-1}[\|A(s, x)(x(s)-y(s))\|+\|(A(s, x)-A(s, y)) y(s)\|] d s \\
& +\frac{1}{\Gamma(\alpha)} \max _{i, k}\left\{1, \prod_{j=i}^{k}\left\|b_{j}\left(\tau_{j}\right)\right\|\right\} \int_{t_{0}}^{t}(t-s)^{\alpha-1}\left\|f\left(s, x_{s}\right)-f\left(s, y_{s}\right)\right\| d s \\
\leq & \frac{1}{\Gamma(\alpha)} \max _{i, k}\{1, B\} \int_{t_{0}}^{t}(t-s)^{\alpha-1}\left[\left(L_{3}\|x\|+k\right)\|x(s)-y(s)\|+L_{3}\|x-y\|\|y(s)\|\right] d s \\
& +\frac{1}{\Gamma(\alpha)} \max _{i, k}\{1, B\} \int_{t_{0}}^{t}(t-s)^{\alpha-1}\left[L_{1}\|x-y\|\right] d s .
\end{aligned}
$$

Therefore,

$$
\begin{aligned}
\sup _{t_{0} \leq t \leq T} E \|(S x(t)-S y(t) \| \leq & \frac{1}{\Gamma(\alpha)} \max _{i, k}\{1, B\} \int_{t_{0}}^{t}\left\{( t - s ) ^ { \alpha - 1 } \left[\left(L_{3} \sup _{t_{0} \leq t \leq T} E\|x\|_{s}+k\right) \times\right.\right. \\
& \left.\left.\sup _{t_{0} \leq t \leq T} E\|x(s)-y(s)\|_{s}+L_{3} \sup _{t_{0} \leq t \leq T} E\|x-y\|_{s} \sup _{t_{0} \leq t \leq T} E\|y(s)\|_{s}\right]\right\} d s \\
& +\frac{L_{1}}{\Gamma(\alpha)} \max _{i, k}\{1, B\} \int_{t_{0}}^{t}(t-s)^{\alpha-1} \sup _{t_{0} \leq t \leq T} E\|x-y\|_{s} d s \\
\leq & \frac{\left(T-t_{0}\right)^{\alpha}}{\Gamma(\alpha+1)} \max \{1, B\}\left[2 L_{3} r+k+L_{1}\right] \sup _{t_{0} \leq t \leq T} E\|x-y\|_{t} .
\end{aligned}
$$

Thus,

$$
\|S x(t)-S y(t)\| \leq\left\{\frac{\left(T-t_{0}\right)^{\alpha}}{\Gamma(\alpha+1)} \max \{1, B\}\left[2 L_{3} r+k+L_{1}\right]\right\}\|x-y\|
$$

Therefore, by Equation (4), $S$ is a contraction.

Step III: To prove that $\mathrm{U}$ is a completely continuous operator.

For that, first we prove that $U$ is uniformly bounded.

For any $t \in\left[t_{0}, T\right]$, consider

$$
\begin{aligned}
\|U x(t)\| \leq & \left\|g\left(t, x_{t}\right)\right\|+\sum_{k=0}^{\infty}\left[\sum_{i=1}^{k}\left\|g\left(t_{i}, x_{t_{i}}\right)\left(b_{i}\left(\tau_{i}\right)-1\right)\right\| \prod_{j=i+1}^{k}\left\|b_{j}\left(\tau_{j}\right)\right\|\right. \\
& \left.+\prod_{i=1}^{k}\left\|b_{i}\left(\tau_{i}\right)\right\|\left\|\phi(0)-g\left(t_{0}, \phi\right)\right\|\right] I_{\left[\zeta_{k}, \zeta_{k+1}\right)}(t)
\end{aligned}
$$


Therefore,

$$
\begin{aligned}
\sup _{t_{0} \leq t \leq T} E\|U x(t)\| \leq & L_{2}+\max _{k}\left\{\sum_{i=1}^{k}\left\|g\left(t_{i}, x_{t_{i}}\right)\left[b_{i}\left(\tau_{i}\right)-1\right]\right\|\right\} \max _{i, k}\left\{\prod_{j=i+1}^{k}\left\|b_{j}\left(\tau_{j}\right)\right\|\right\} \\
& +\max _{k}\left\{\prod_{i=1}^{k}\left\|b_{i}\left(\tau_{i}\right)\right\|\right\} \sup _{t_{0} \leq t \leq T} E\left\|\phi(0)-g\left(t_{0}, \phi\right)\right\| \\
\leq & L_{2}+B\left(N+\sup _{t_{0} \leq t \leq T} E\left\|\phi(0)-g\left(t_{0}, \phi\right)\right\|\right)
\end{aligned}
$$

That implies that, $\|U x(t)\| \leq L_{2}+B\left(N+\left\|\phi(0)-g\left(t_{0}, \phi\right)\right\|\right)$.

This yields that $U$ is uniformly bounded.

Next, we have to show that $\left\{U x: x \in B_{r}\right\}$ is equicontinuous.

Let $x \in B_{r}$ and let $t_{0} \leq t_{1}<t_{2} \leq T$, then we have

$$
U x\left(t_{2}\right)-U x\left(t_{1}\right)
$$

$$
\begin{aligned}
= & {\left[g\left(t_{2}, x_{t_{2}}\right)+\sum_{k=0}^{\infty}\left[\sum_{i=1}^{k} g\left(t_{2 i}, x_{t_{2 i}}\right)\left(b_{i}\left(\tau_{i}\right)-1\right) \prod_{j=i+1}^{k} b_{j}\left(\tau_{j}\right)+\prod_{i=1}^{k} b_{i}\left(\tau_{i}\right)\left[\phi(0)-g\left(t_{0}, \phi\right)\right]\right] I_{\left[\zeta_{k}, \zeta_{k+1}\right)}\left(t_{2}\right)\right] } \\
& -\left[g\left(t_{1}, x_{t_{1}}\right)+\sum_{k=0}^{\infty}\left[\sum_{i=1}^{k} g\left(t_{1 i}, x_{t_{1 i}}\right)\left(b_{i}\left(\tau_{i}\right)-1\right) \prod_{j=i+1}^{k} b_{j}\left(\tau_{j}\right)+\prod_{i=1}^{k} b_{i}\left(\tau_{i}\right)\left[\phi(0)-g\left(t_{0}, \phi\right)\right]\right] I_{\left[\zeta_{k}, \zeta_{k+1}\right)}\left(t_{1}\right)\right] \\
= & g\left(t_{2}, x_{t_{2}}\right)-g\left(t_{1}, x_{t_{1}}\right)+\sum_{k=0}^{\infty}\left[\sum_{i=1}^{k} g\left(t_{2 i}, x_{t_{2 i}}\right)\left(b_{i}\left(\tau_{i}\right)-1\right) \prod_{j=i+1}^{k} b_{j}\left(\tau_{j}\right)\right. \\
& \left.+\prod_{i=1}^{k} b_{i}\left(\tau_{i}\right)\left[\phi(0)-g\left(t_{0}, \phi\right)\right]\right]\left[I_{\left[\zeta_{k}, \zeta_{k+1}\right)}\left(t_{2}\right)-I_{\left[\zeta_{k}, \zeta_{k+1}\right)}\left(t_{1}\right)\right] \\
& +\sum_{k=0}^{\infty}\left[\sum_{i=1}^{k}\left[g\left(t_{2 i}, x_{t_{2 i}}\right)-g\left(t_{1 i}, x_{t_{1 i}}\right)\right]\left(b_{i}\left(\tau_{i}\right)-1\right) \prod_{j=i+1}^{k} b_{j}\left(\tau_{j}\right)\right] I_{\left[\zeta_{k}, \zeta_{k+1}\right)}\left(t_{1}\right)
\end{aligned}
$$

Then

$$
\left\|U x\left(t_{2}\right)-U x\left(t_{1}\right)\right\| \leq\left\|g\left(t_{2}, x_{t_{2}}\right)-g\left(t_{1}, x_{t_{1}}\right)\right\|+\left\|I_{1}\right\|+\left\|I_{2}\right\|
$$

where,

$$
\begin{aligned}
E\left\|I_{1}\right\| \leq & E\left(\max _{k}\left\{\sum_{i=1}^{k}\left\|g\left(t_{2 i}, x_{t_{2 i}}\right)\left(b_{i}\left(\tau_{i}\right)-1\right)\right\|\right\} \max _{i, k}\left\{\prod_{j=i+1}^{k}\left\|b_{j}\left(\tau_{j}\right)\right\|\right\}\right. \\
& \left.+\max _{k}\left\{\prod_{i=1}^{k}\left\|b_{i}\left(\tau_{i}\right)\right\|\right\}\left\|\phi(0)-g\left(t_{0}, \phi\right)\right\|\left[I_{\left[\zeta_{k}, \zeta_{k+1}\right)}\left(t_{2}\right)-I_{\left[\zeta_{k}, \zeta_{k+1}\right)}\left(t_{1}\right)\right]\right) \\
\leq & B\left(N+E\left(\left\|\phi(0)-g\left(t_{0}, \phi\right)\right\|\right)\right) E\left(I_{\left[\zeta_{k}, \zeta_{k+1}\right)}\left(t_{2}\right)-I_{\left[\zeta_{k}, \zeta_{k+1}\right)}\left(t_{1}\right)\right) \\
\rightarrow 0{\text { as } t_{2} \rightarrow t_{1}} &
\end{aligned}
$$




$$
\begin{aligned}
& E\left\|I_{2}\right\| \leq E\left(\max _{k}\left\{\sum_{i=1}^{k}\left\|\left[g\left(t_{2 i}, x_{t_{2 i}}\right)-g\left(t_{1 i}, x_{t_{1 i}}\right)\right]\left(b_{i}\left(\tau_{i}\right)-1\right)\right\|\right\}\right. \\
&\left.\max _{i, k}\left\{\prod_{j=i+1}^{k}\left\|b_{j}\left(\tau_{j}\right)\right\|\right\} I_{\left[\zeta_{k}, \zeta_{k+1}\right)}\left(t_{1}\right)\right) \\
& \leq B \max _{k}\left\{\sum_{i=1}^{k} E\left(\left\|\left[g\left(t_{2 i}, x_{t_{2 i}}\right)-g\left(t_{1 i}, x_{t_{1 i}}\right)\right]\left(b_{i}\left(\tau_{i}\right)-1\right)\right\|\right)\right\} \\
& \rightarrow 0 \text { as } t_{2} \rightarrow t_{1}
\end{aligned}
$$

From the Equations (6) and (7), the right hand side of the Equation (5) $\rightarrow 0$ as $t_{2} \rightarrow t_{1}$.

$$
\left\|U x\left(t_{2}\right)-U x\left(t_{1}\right)\right\|=\sup _{t_{0} \leq t \leq T} E\left\|U x\left(t_{2}\right)-U x\left(t_{1}\right)\right\| \rightarrow 0
$$

as

$$
t_{2} \rightarrow t_{1}
$$

Thus, $U$ is equicontinuous.

\section{Example}

Let $\tau_{k}$ be a random variable defined in $D_{k} \equiv\left(0, d_{k}\right)$ for all $k=1,2, \ldots$ where $0<d_{k}<\infty$. Furthermore, assume that $\tau_{i}$ and $\tau_{j}$ be independent with each other as $i \neq j$ for $i, j=1,2, \ldots$

Consider, the following fractional differential equation with random impulses of the form:

$$
\left\{\begin{array}{l}
{ }^{c} D^{\alpha}\left(x(t)-\frac{\cos t}{(t+3)^{2}} \frac{x}{1+x}\right)=\frac{1}{9} \sin x(t) x(t)+\frac{1}{t+1} \frac{x}{x+9}, \quad t \in\left[t_{0}, T\right], t \neq \zeta_{k} \\
x\left(\zeta_{k}\right)=p(k)\left(\tau_{k}\right) x\left(\zeta_{k}^{-}\right), \quad k=1,2, \ldots \\
x_{t_{0}}=\phi
\end{array}\right.
$$

It is easily seen that the functions $f, g$ and $A$ satisfies the assumptions and clearly, we have $L_{1}=L_{2}=L_{3}=k=\frac{1}{9}$.

Moreover the assumptions $\left(H_{5}\right)$ and $\left(H_{6}\right)$ are satisfied.

Further, if $r=1$, from the above facts, in view of Theorem (3), we conclude that the Equation (8) has a solution on $\left[t_{0}, T\right]$, provided that the inequalities:

$$
\frac{4\left(T-t_{0}\right)^{\alpha}}{9 \Gamma(\alpha+1)} \max \{1, B\}+\frac{1}{9}+B\left(N+\left\|\phi(0)-g\left(t_{0}, \phi\right)\right\|\right) \leq 1
$$

and

$$
\frac{4\left(T-t_{0}\right)^{\alpha}}{9 \Gamma(\alpha+1)} \max \{1, B\}<1
$$

are satisfied. 


\section{Acknowledgments}

This work has been supported in part by the Government of Spain and FEDER (grant No. MTM2013-41704-P) and by University Grants Commission,India(grant No. 41-780/2012(SR)).

\section{Author Contributions}

All authors have contributed equally.

\section{Conflicts of Interest}

The authors declare no conflict of interest.

\section{References}

1. Kilbas, A.A.; Srivastava, H.M.; Trujillo, J.J. Theory and Applications of Fractional Differential Equations; Elsevier Science Limited: Amsterdam, the Netherlands, 2006.

2. Podlubny, I. Fractional Differential Equations; Academic Press: Waltham, MA, USA, 1993.

3. Balachandran, K.; Trujillo, J.J. The nonlocal Cauchy problem for nonlinear fractional integrodifferential equations in Banach Spaces. Nonlinear Anal. Theory Methods Appl. 2010, $72,4587-4593$.

4. Diethelm, K. Analysis of fractional differential equations. J. Math. Anal. Appl. 2002, 265, 229-248.

5. Miller, K.S.; Ross, B. An introduction to the fractional calculus and fractional differential equations; Wiley: Hoboken, NJ, USA, 1993.

6. Lakshmikantham, V.; Leela, S.; Vasundhara Devi, J. Theory of Fractional Dynamic Systems; Cambridge Scientific Publishers: Cambridge, UK, 2009.

7. Lakshmikantham, V.; Vatsala, A.S. Basic theory of fractional differential equations. Nonlinear Anal. 2008, 69, 2677-2682.

8. Hernandez, E.; O'Regan, D.; Balachandran, K. On recent developments in the theory of abstract differential equations with fractional derivatives. Nonlinear Anal. Theory Methods Appl. 2010, 73, 3462-3471.

9. Liu, Y.C.; Wu, J.; Li, Z.X. Multiple solutions of some impulsive three point boundary value problems. Dyn. contin. Discrete Impuls. Syst. 2006, 13A, 579-586.

10. Lakshmikantham, V.; Bainov, D.D.; Simeonov, P.S. Theory of Impulsive Differential Equations; World Scientific: Singapore, 1989.

11. Anguraj, A.; Vinodkumar, A. Existence and uniqueness of neutral functional differential equations with random impulses. Int. J. Nonlinear Sci. 2009, 8, 412-418.

12. Wu, S.; Guo, X.-L.; Lin, S.-Q. Existence and uniqueness of solutions to random impulsive differential systems. Acta Math. Appl. Sin. 2006, 22, 627-632.

13. Wu, S.J.; Duan, Y.R. Oscillation, stability and boundedness of second-order differential systems with random impulses. Comput. Math. Appl. 2005, 49, 1375-1386. 
14. Anguraj, A.; Vinodkumar, A. Existence, uniqueness and stability results of random impulsive semilinear differential systems. Nonlinear Anal. Hybrid Syst. 2010, 3, 475-483.

15. Anguraj, A.; Wu, S.; Vinodkumar, A. The existence and exponential stability of semilinear functional differential equations with random impulses under non-uniqueness. Nonlinear Anal. 2011, 74, 331-342.

16. Benchohra, M.; Slimani, B.A. Existence and Uniqueness of solutions to impulsive fractional differential equations. Electron. J. Differ. Equ. 2009, 10, 1-11.

17. Benchohra. M.; Seba, D. Impulsive fractional differential equations in Banach Spaces. Electron. J. Qual. Theory Differ. Equ. Spec. Ed. I 2009, 8, 1-14.

18. Chuanxi, Q.; Ladas, G. Oscillations of first order neutral equation with variable coefficients. $M h$. Math. 1990, 109, 103-111.

19. Gopalsamy, K. Oscillations in neutral delay differential equations. J. Math. Phys. Sci. 1990, 21, 23-34.

20. Grove, E.A.; Kulenovic, M.R.S.; Ladas, G. Sufficient conditions for oscillations and non-oscillations of neutral equations. J. Differ. Equ. 1987, 68, 673-682.

21. Agarwal, R.P.; Zhou, Y; He, Y.Y. Existence of fractional neutral functional differential equations. Comput. Math. Appl. 2010, 59, 1095-1100.

22. Anguraj, A.; Ranjini, M.C. Existence results for fractional impulsive neutral functional differential equations. J. Fract. Calc. Appl. 2012, 3, 1-12.

(c) 2015 by the authors; licensee MDPI, Basel, Switzerland. This article is an open access article distributed under the terms and conditions of the Creative Commons Attribution license (http://creativecommons.org/licenses/by/4.0/). 